\title{
Advantage of Early Tangential Necrectomy in Extensive Pediatric Burns.
}

\author{
Kenan Karavdić 1 \\ https://doi.org/10.32391/ajtes.v3i2.47
}

\begin{abstract}
Background: An extensive burn is one of the most serious injuries. The conventional methods of local therapy, especially in extensive and deep burns, cannot prevent infection. The fact is that the necrotic burn tissue always gives rise to infection and must therefore be excised. Tangential excision is a diagnostic procedure as well as a surgical preparation of a recipient bed for skin graft.

Aim of research: The aim of our research was to demonstrate the efficacy of early tangential necrectomy in the treatment of extensive burns in children as well as in preventing the burn diseases.

Material and methods: Clinical parameters of sixty extensively burned children have been evaluated, divided into two groups (surgically and non-surgically treated) and in four subgroups (under and over the 25\% TBSA). The following criteria have been used to compare the results: pain, fever, body weight, duration of the hospital stay, wound infection, laboratory values.

Results: The boys comprised $58.3 \%$ of the respondents and the girls were $41,7 \%$ of the respondents. The most common cause was the hot liquid of $73.3 \%$, open flame $23.3 \%$ and the contact with hot metal $3.3 \%$. The body temperature was significantly higher in control group compared to the examined group ( $p$ $<0.001)$ significantly lower hematocrit values in the control group compared to the examined group $(\mathrm{p}=$ 0.002). The results show that hospitalization lasted considerably longer in patients in the control group than in the examined group $(\mathrm{p}=0.003)$.

The incidence of bacterial infection of the burns is significantly higher in control compared to the examined group of patients $(\mathrm{p}=0.007)$. The most commonly isolated bacteria in the study group was Staphylococcus aureus (15/30 or $50 \%$ of all subjects). Acinetobacter spp. was isolated in 9 patients ( $30 \%$ of respondents). Serratia spp. And Enterococcus Foecalis were isolated in 6 patients $(20 \%)$. Pseudomonas aeruginosa was isolated in 3 patients $(10 \%)$. Three patients $(10 \%)$ did not isolate any bacteria during hospitalization Conclusions: The method of early tangential necrectomy of extensively burned children significantly reduce morbidity and mortality (it improves the general condition of the patient, improves clinical and laboratory parameters, reduces the possibility of infection of the wound, reduces the possibility of sepsis, reduces the length of hospitalization). The most optimal time to perform an early tangential necrectomy is the period between the 4th and the 6th day after the injury. In extensively burned children over $30 \%$ of the total body surface, an early tangential necrectomy should be performed on an area of about $20 \%$ of the total body surface as a prevention of blood loss due to necrectomy and autotransplantation. With extensively burned children over $40 \%$ of the total body surface, taking homotransplants from parents or close relatives reduces morbidity and mortality.

Keywords: pediatric, burns, extensive
\end{abstract}

${ }^{*}$ Corresponding Author: Kenan Karavdić

$=凶$ E-mail: kenan.kv@bih.net.ba

${ }^{1}$ Clinic for Pediatric Surgery, Clinical Center of University Sarajevo, Sarajevo, Bosnia and Herzegovina 


\section{Full Text}

\section{Introduction}

Extensive burns are especially important in trauma because of their common lethal outcome and physical, sensory and aesthetic sequelae. The most common causes of burns are fire, hot water, hot vapors, flammable liquids and gases. Recent statistics show that mortality rate in United States is 57.1 per 1000000 and in Canada 29.7 per 1000000 population (1).

The heat effect on the cell depends of the temperature and the period or temperature is acting. Moritz and Henriquez were among the first to create a weather-temperature relationship for thermal injuries. Exposure to temperatures between 40 and $44 \mathrm{C}$ leads to weakening of function and early protein denaturation (2). Damage to the membrane is also impaired by cellular function, resulting in high intracellular concentration and subsequent edema. When the temperature is $44 \mathrm{C}$ or higher, damage is overridden by the insertion cell repair mechanism, the exposure time is long enough that the significant modified plasma membrane is sufficient to cause the necrosis. The damage that occurs is characterized by the participation of free radical oxygen, highly reactive molecules capable of promoting the future abnormalities of the cell membrane, leading to the death of the cell.

Further growth of temperature increases the protein destruction. Early protein denaturation destroys all levels of protein architecture and new aberrant links are formed, creating macromolecules that do not resemble the original structures. Necrosis of cells is complete, usually initiated on the skin surface where the heat energy is most directly received, extending deeper.

Coagulation is a zone of necrosis and is the first of three zones described by Jackson (3), which includes the initial bladder eschara.

- The zone of stasis lies deeper and more peripheral than the coagulation zone and represents a minor area. The development of ischemia in this zone may devastate already compromised cells, in some cases it can turn the entire zone into a dead eschara. Blood flow damage begins with microvascular events including platelet microtrombosis, adhesion of neutrophils to the walls, fibrin retention, and vasoconstriction (4.5). In optimal conditions, the zone of stasis can be reversible with cells which heal within one week. During the period of healing, the tissues are very fragile and suppressive to additional insults.

Zone of hyperemia is peripheral, characterized by minimal cell injury but with prominent vasodilation, with increased blood flow during which vasoactive mediators were produced as part of the inflammatory response. This zone usually prefers complete cellular healing except complications such as infection or trauma. 


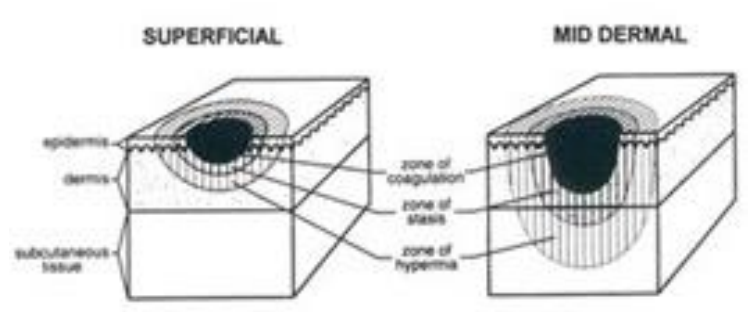

Figure 1: Zone of coagulation

Eschara represents an open wound where the protective role of the skin is lost. In the beginning, the eschara shortly helps the barrier: mechanical, immunological, and antibacterial, in fact, allows the environment to increase bacteria $(6,7)$. This problem may be greater by taking water or targeted aplicated drugs from the surrounding tissue by the eschara. The ability of the skin to prevent loss of liquidity, electrolyte, and plasma components has disappeared and the eschara has therefore a high degree of permeability. It has been shown that high levels of loss of protein through the eschara even after the decrease of capillary permeability (8). The loss of complement and immunoglobulin components is increased by burn-induced immune suppression, while protein loss generally damages nitrogen balance and wound healing. Eschara may play a role in the hemostasis damage (platelets, fibrinogen) over time. The thermoregulatory role provided by normal skin also disappeared into the eschara. Even the eschara tissue is metabolically dead and contains heat derivatives and toxins that are diffuse to circulation, causing dysfunction of distant organs and other problems.

The loss of intravascular fluid and protein is caused by heat-damaged capillaries. There is great permeability between endothelial cells, which even allows macromolecules, such as fibrinogen, to pass through the interstitium.

By definition, edema develops when the value by which the liquidity is filtered out of the microsurgies exceeds the flow in the lymph nodes by draining the tissue mass. Edema formation appears biphasic. In the first few hours after the injury, a sudden increase in fluid flow of the ingested tissue occurs. The second and much larger increase in fluid loss and unprotected tissue is followed in the first 12 to 24 hours after the injury to maintain a high level of $48-72$ hours. The size of edema of the cut skin depends on the type and extent of the burn and the time when the restitution fluid is given. The degree of burn injury depends on the following factors (1) depth, and location; (2) patient age; (3) the etiology of the agent causing it; (4) the presence of inhalation injuries; and (5) associating with persistent illness. The American Burn Association has used these parameters in determining the guide to the classification of burn weight. Classification has created 3 categories of burn injuries (large-extensive, medium, small) and defined the optimal framework for each bladder treatment. Determination of the percentage of the cleaved surface is done after primary treatment using a Lund-Browder Modified Child Modification for Children, which was accepted in the International Classification of Burns. 


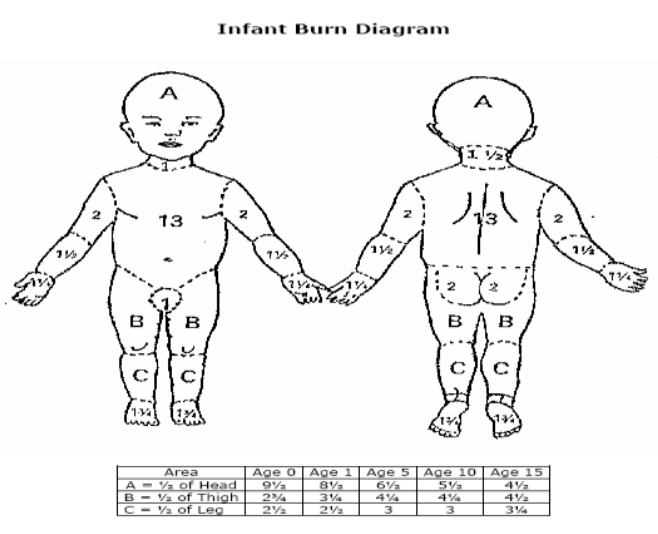

Figure 2: Lund-Brower-diagram

\section{Burn disease (infection of burns and sepsis)}

Extensive burns have a higher risk of infection. Infection can lead to generalized disease (burn toxemia) by absorbing toxic products produced by the development of bacteria, leading to the cause and represents the most common and most serious complication of extensive burns. The area of the swelling wound is sterile immediately after the burn, but in the first $48 \mathrm{~h}$ it encounters gram positive organisms from hair follicles, skin adnex and the environment. Much more virulent gramnegative organisms replace grampositive organisms after 5-7 days. Gramnegative organisms have greater mobility, possess many mechanisms of resistance to antibiotics, and have the ability to capture collagenases, proteases, lipases and elatases, enabling them to penetrate the sub-area. If the defense is inadequate, the invasion of vital tissue continues. When the number of bacteria is $>10 * 5 / \mathrm{g}$ risk of early infection is large. If the number of bacteria is less than $10 * 3 / \mathrm{g}$, the bacteria is usually noninvasive. The most common and most dangerous bacteria that cause burn infections are Pseudomonas, Proteus and Staphylococcus aureus ("The Devil's Trio"). Usually it is resistant to all antibiotics and chemotherapy.

Sepsis is usually clinically manifested by bronchopneumonia, pyelonephritis, thrombophlebitis or invasive infection of the wound. The burn wound is an ideal substrate for bacterial growth and allows a large gateway to bacterial invasion. Microbiotic colonization of open burn wounds, a primary endogenous source, was established by the end of the first week. Infection is enabled by the loss of epithelial barriers induced by malnutrition as a hypermetabolic response to burn injury and generalized post-ophthalmic immunosuppression by releasing immunoreactive agents from the burn-on wound.

The burn wound causes suppression of almost all aspects of the immune response. Post-burn levels of immunoglobulin, fibronectin, and level of complement are reduced. Hemotaxis, phagocytosis, the role of neutrophils, monocytes and macrophages are damaged. Granulocytopenia is common in burns. The cellular immune response, recorded in the late reaction to the alograft, was damaged by lymphocyte mitogenesis. The burn results in a reduction in interleukin-2 (II-2) production, cytotoxic T-cell and NK cells, and helps T-cell (HSR) suppression. During the whole process of demarcation of dermal burns, there are severe symptoms of the disease and "burning illness" in the patient. Septic symptoms are the reflection of an 
inflammatory reaction that develops in a large portion of dermal tissue rich in vascular network.

"Primary pain" caused by direct nausea is followed by a "secondary pain" resulting from intradermal pressure increase. Poor general condition, high temperature, anorexia and severe pain are present in patients as well as rapid fall in blood protein levels and hemoglobin.

\section{Reanimation and initial treatment of the acquired}

Burn shock is also a hypovolemic and cellular shock and is characterized by specific hemodynamic changes, including heart rate reduction, extracellular plasma and oliguria. As with other forms of shock therapy, the primary goal is to maintain tissue perfusion and prevent tissue ischemia. In case of burning shock, reanimation is complicated due to mandatory bladder edema.

The first studies by Frank P.Underhill, made 100 years ago, were investigated by fire victims at Rialto Theater in 1921 (9). His concept of burning shock due to the loss of intravascular fluid was further illuminated by Moor who added to the major Coconut Grove patients 1942 (10). They developed the concept of burning edema and presented a body weight formula for reanimating fluid-trained patients. In 1952, Artz and Moncrief developed a surface-to-weight bladder formula for fluid resuscitation, which became the first resuscitation formula for resuscitation of acquired patients (11). Evans' formula was introduced in 1952, and the Brooke Army Medical Center modified the original Evans formula, which became standard for the next 15 years.

Reanimation with crystalline, especially Ringer lactate solute with $130 \mathrm{mEq} / 1$ sodium, is the most popular reanimation fluid. The authors only use of the resilience crystalline solution have revealed that other liquids, especially colloids, are not better and much more expensive than crystalloids (12). The most common reason given for the lack of colloid is that even large proteins pass through the capillaries after a thermal injury. The amount of crystalloid needed depends on the parameters of reanimation monitoring. The Perkland formula recommends $4 \mathrm{ml} / \mathrm{kg} / \%$ of the cleaved surface in the first 24 hours, with the half administered in the first 8 hours (13). Modified Brooke formula recommends the beginning of reanimation with $2 \mathrm{ml} / \mathrm{kg} / \%$ in the first 24 hours.

Reanimation by hypertonic $\mathrm{NaCl}$, first published by Monafo, is the treatment of burn victims with saline solution 240-300 $\mathrm{mEq} / 1$, resulting in lower edema due to minor needs (14). The Burn Children Institute in Cincinnati uses a modified hypertonic solution for large burns of over $40 \%$. Reanimation fluid contained $180 \mathrm{mEqNa}$ (Ringer lactate $+50 \mathrm{mEq}$ $\mathrm{NaHCO} 3)$. The solution was used while metabolic acidosis occurred, usually 8 hours after burns. The volume was initially administered by the amount calculated for the Perkland formula $(4 \mathrm{ml}$ / $\mathrm{kg} / \%$ burn), volume shifted to diuretic $30-50 \mathrm{ml} / \mathrm{h}$. The hypertensive formula can be used in neonates without the risk of hypernatriemia (15). 


\section{Reanimation with colloids}

Evans 1952, uses $1 \mathrm{ml} / \mathrm{kg} / \%$ of burns and for colloids and Ringer lactate over the first 24 hours, and Brooke formula uses $0.5 \mathrm{ml} / \mathrm{kg} / \%$ of Colombo bladder and $1.5 \mathrm{ml} / \mathrm{kg} /$ lactate. There are three schools and opinions for the use of proteins in reanimation formulas:

1) Protein solutions should not be given for the first 24 hours because during this period they are not very effective while salt water maintains the intravascular volume.

2) Proteins, especially albumin, should be given from the beginning of reanimation along with the crystalloids, they are usually added to salt water.

3) Proteins should be given between 8 and 12 hours after burns using strict crystalloids in the first 8 to 12 hours due to massive fluid loss in that period.

Demling experimentally demonstrates that plasma protein maintenance is not effective for the first 8 hours after burns (16).

In young patients with large burns, recurrence of colitis often occurs because of the sudden drop in the concentration of serum proteins in burn shock.

The volume required to compensate patient depends on the type of injury, age, physiological status and related injuries. Consequently, the volume is predisposed to the substitution formula with the most commonly modified individual response to therapy. Using useful properties of hypertonic solutions, crystalloids and colloids at different times after burns, will reduce the amount of edema. The level of administration of the compensated fluid should be maintained by diuretics of $30-50 \mathrm{ml}$ in adults and $1-2 \mathrm{ml} / \mathrm{kg}$ in children. When the weight of children is $30-50 \mathrm{~kg}$, diuresis should be maintained at the adult level. Serial Serum Concentration is not the only route of dehydration diagnosis but is the best guide to planning and tracking successful fluid replenishment. Other laboratory useful hydration indicators and guides include: Weight change, Sodium levels in serum and urine (concentration), Sugar (concentration in serum and urine), Input and Output, and Clinical Trials. Permanent colloid remission may be necessary to maintain colloidal oncotic pressure in very large burns and children patients. The maintenance of serum albumin levels of about $40 \mathrm{~g} / \mathrm{dl}$ is desirable. Electrolyte $\mathrm{Ca}, \mathrm{Mg}$ and $\mathrm{P}$ can also be monitored. Requires $0.3-6.5 \mathrm{ml}$ / $\mathrm{kg}$

Children have limited physiological reserves. The Shriner Burns Institute has demonstrated that children with burns need more fluids for the reanimation then adults (the need for fluids for children is $5.8 \mathrm{ml} / \mathrm{kg} / \%$ of the treated area) (17). Children usually need intravenous replacement for relatively small burns of $10-20 \%$ of the body surface. Baxter publised similar needs for a pediatric age group (35/Graves and association demonstrated that children received $6.3+-2 \mathrm{ml} / \mathrm{kg} / \%$ of burns (18). The Shriners Burns Institute, Cincinnati Unit, uses a Parkland formula with an addition of $4 \mathrm{ml} / \mathrm{kg} / \%$ burn in $24 \mathrm{~h}+$ $1500 \mathrm{ml} / \mathrm{m} 2$.

\section{Local treatment of burns}

The aim of local burn wound treatment is the prevention of vital tissue 
involvement and bacterial control. The burn wound covered with serum exudate is the ideal surface for uncontrolled growth of bacteria. Infection is the most important cause of so-called "burning toxemia". The most common and most dangerous bacteria that cause burn infection are: Pseudomonas, Proteus and Staphylococcus aureus are usually resistant to all antibiotics and chemotherapy.

Local treatment consists of local hemoprophylaxis (antiseptic methods) and systemic use of antibiotics against colonizing colonies. Antimicrobial agents and biological bands are applied locally.

-Silver nitrate $0.5 \%$ is effective for Pseudomonas aeruginosa and Proteus, less effective on Staphylococcus aureus. -Silver sulfadiazine is the most commonly used local antimicrobial agent for burns. It covers gram positive and most gram negative bacteria. Unlike Silver Nitrate, it does not prevent epithelialization and prevents fibroblast contraction.

Specifity of pediatric extensive burns :

- The body surface area in children compared to the body surface is much higher than in adults.

- The blood pool is smaller in children than in adults.

- Underdeveloped kidneys and livers can not withstand an increased burden of excretion and adequate nutritional needs and support. Differences in renal function between infants and adults may have important therapeutic implications in the treatment of premature infants. The degree of glomerular filtration does not reach the level of adults up to 9-12 months due to unbalance in tubular and glomerular function maturation. During this period, newborn babies have at most half the osmolar capacity of the adult.

- Insufficiently developed immune system increases the possibility of infection.

\section{Early tangential necrotomy}

Reverdin first introduced the epidermal graft and Thiersch in 1874 used more extensively skin parts. Wolf used a free full graft to reconstruct the lower eyelid defect. In 1891 Lustgarten described the technique of early excision and covering the graft of minor burns. Alografts have also been used for many years in the treatment of burns. Brown from St.Louis has established practical aspects of biological coverage as a treatment of extensive burns.

However, the first serious investigations of the surgical scarring treatment was in 1929 when Blair stated that the burning necrosis should be excreted and covered as soon as possible with the graft. The first results in that heroic surgery were described in 1960 by D.Jackson, B.G.MacMillan and W.Altemeir, who presented a small advantage over surgical conservative treatment. Soon after, R.MacCormack modified this procedure by separating the excision and covering the graft in a few days. S.R.Lewis has published experience with a series of patients where time-telling and graft coverage were not before $24 \mathrm{th}$ day after burns. The method of tangential necrectomy was introduced by D.M.Jackson and Z.Janžekovič separately as the right time for operative 
treatment, is the period of the $3 \mathrm{rd}$ to the 5 th day after the injury, and introduces the term "wound" for the first time.

The term tangential excision implies excision of the necrotic surface of the burn by repeated parallel slices using a skin graft knife. This method contradicts the earlier method of exfoliation of fatty tissue or deep fasciation by scalpel, exfoliating skin and subcutaneous tissue together. The procedure involves tangential excision and grafting. The goal of the procedure is to apply early closure of extensive burns. This surgical method treats deep dermal burns. By this method, the possibility of damage to undetected dermis is smaller in relation to the previously applied excision method of necrotic mass. Tangential necrectomy, correctly performed, can not transform partial into the entire skin thickness because the deepest quarter of the skin does not excel with the glands of the stinging. Tangential excision is a good diagnostic procedure. The first task is to determine Blair's knife depth of scarring with careful and gradual horizontal excision, after which the island of devitalized tissue is removed by repeated excision in regions where the burn is deeper. Fields showing blood thrombosis are considered potentially necrotic and are therefore completely excreted. Profuse bleeding is a real indicator of skin vitality

The excision of the necrotizing surface is best to start from the center of the burn where it is most likely to be the deepest. The first goal is to find out whether the subdermal veins are thrombosed where the burn is the deepest. If they are present, they will be easily visible after the first bruising layer tangentially excised. If no vein is visible, the case corresponds to tangential necrectomy and covers the vulnerable surface of the grafts. If thrombotic veins are present, the excision should be at the edge of the analgesic region, showing how many of the thrombosed veins and dead fatty tissues are involved, then thrombosed veins and dead fatty tissue can be excised with the scalpel.

The area of hyperemia, located on the red edge of the burn, should not be excreted and it is cured within 7 days of the initiated treatment.

Successively removing the slices from the same region of necrotic tissue should be represented until a pink derm has multiple bloody spots. When the region is noticed as pink and bloody, excision is interrupted even though a few minutes later it looks quite white and avascular. If the small pieces of yellow-brown dermis remain in the center of the pink dermis when the bleeding is stopped, it means that the excision was inadequate and that another layer had to be removed to release the vital substrate. The presence of black, thrombosed subdermal veins shows that fatty tissue is affected and that more tissue has to be excreted. Burning depth is demonstrated and confirmed by consecutive layers of white or pink necrotic skin that has been removed.

Leading skin graft knives such as Braithwaite, Watson or Cobbett modified Humby and Blair knife are recommended for exfoliation. If a knife is satisfactorily fitted, 4 or 5 slices of cut skin can be taken in the area where the skin is thicker. Powerfully powered electric dermatitis, useful for skin grafts, 
is not such a good tool for finding the right dermis thickness.

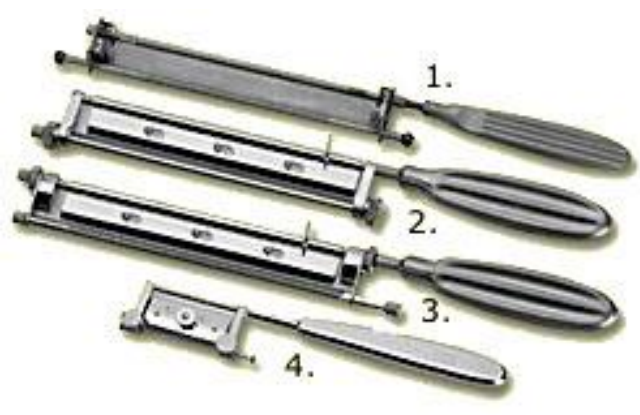

Figure 3: Humby and Blair knives

The burn wound is the source of all ill effects, local and systemic. Eschara is essentially an open wound and a major cause of wound healing. It is also a remarkable environment for the development of bacteria, fluid loss, electrolyte and serum proteins. Eschara plays a role in hemostasis (platelets and fibrinogen) damage. Thrombosis that affects the blood vessels in the pathway compromises the already damaged tissue vitality and causes further damage and development of the infection. Necrosis gradually penetrates into the deeper layers of the skin, forming microabscess that represents the demarcation between vital and necrotic tissue. The infection destroys follicles of hair and a large number of sebaceous glands and sweat glands. Such a necrotizing enviroment is suitable for the development of infection. The next effect of the infection is the destruction of the deep dermis layer and the transformation of dermal burns into the bruising of the stage III. (20) In the case of extensive burns, there is a higher risk of infection. The tissue eschara is metabolically dead and contains heat derivatives and toxins that are diffuse to the circulation causing distal organs dysfunction. (21) Absorption of toxic products produced by the development of bacteria can lead to generalized disease (bovine toxemia) leading to the disease and is one of the major causes of mortality of extensively burnt patients. The most common cause of Pseudomonas aeruginosa and other grams can be represented (Staphylococcus aureus and Streptococcus pyogenes). $(22,23,24)$

Local conservative treatment is based on the treatment of infections and by means of preventative preventive measures (first line of defense) and the means that protect tissue and blood components from bacterial invasion. In the first line of defense we include the use of local hemoprophylaxis or antiseptic methods. The use of $0.5 \%$ of silver nitrate, which shares bactericidal and nonhybridisation, is efficiently removed in case of extensive bacterial bruising such as Pseudomonas aeruginosa and Proteus, while Staphylococcus is less efficiently controlled and on different coliform bacilli weak or ineffective. (23) Surgical treatment of extensive burns (deep or level III) involves removing the wound (avascular lesion) and converting it to the uncovered untreated surgical wound and then covering that region with some of the grafts. This eliminates necrotic lactate-rich tissue, improves collagen synthesis, reduces the activity of inflammatory mediators and their contribution to microcirculation and permeability of blood vessels, reduces protein loss, and reduces the risk of dysthymia. All this leads to improvement of the general condition of 
the patient and reduction of hospital stay. $(20,25,26)$.

The authors of D.M.Jackson and Z.Janžekovič believe that the demarcation between vital and ireversibly destroyed (necrotic) tissues becomes apparent after the first phase of burn-out shock characterized by coagulant necrosis, dilated capillaries and increased blood vessel permeability. In the second stage, the organism reacts to the necrosis of the inflammatory reaction that may cause secondary necrosis of the deeper, vital layers of the dermis with microtrombosis. Taking into account that during this period (first 3 days) the resuscitation was completed, the reanimation carried out, the tissue of the diseased tissue, the authors considered that the period from the 3rd to the 5th day after the injury was the most ideal for carrying out the operative procedure.

\section{Aim of study}

The aim of our research was to demonstrate the efficacy of early tangential necrectomy in the treatment of extensive burns in children as well as in preventing the burns diseases.

\section{Material and methods}

In accordance with the objectives of the study, a homogeneous sample of respondents was chosen to reduce the polyciability of observed parameters.

The group consisted 30 children ages 6 months to 14 years, with extensive burns treated with surgical, early tangential necrectomy with mash autograft at the KCU Sarajevo Children's Clinic.

The control group consisted of 30 children ages 6 months to 14 years, with extensive burns treated with a conservative treatment method and children treated with surgical, delayed tangential necrectomy at the $\mathrm{KCU}$ Sarajevo Children's Clinic.

In order to analyze the impact of the body surface area on the length of the treatment, the subjects were divided into 2 subgroups, where the first subgroup consisted of children up to $25 \%$ and the other children over $25 \%$ of the body surface.

The study was conducted on the basis of a common approach to a patient, an anamnesis and a clinical examination. The pediatric surgeons have completed questionnaires that contain general information about the child (name, surname, full date of birth etc.), previous disease data, injury data that includes the agent of injury, time and place of injury, transport, first aid ... Clinical overview included the determination of the total area of burn, classification of burns according to the American Society for Burns with categorization. Thereafter, a reanimation with Perkland's formula was performed recommended by Shriners Burns Institute Cincinnati Unit, following clinical parameters (body weight, temperature, diuresis) and laboratory findings (hemogram, proteinogram). Patients were conservatively treated, and on the 3rd and 7th day after the injury, if identified by clinical examination of necrotic changes, it was applied early tangential excision with mash-autograft coverage. After the performed procedure, the 
clinical and laboratory follow-up of the patient continued. Certainly, the survival and duration of treatment in the hospital was also monitored.

Patients with previous metabolic and hematological diseases and patients with death were excluded from the study.

\section{Operative procedure}

Early tangential necrectomy with autotransplantation of mash-graft in all subjects was performed at the Clinic for Child Surgery of the Clinical Center of the University of Sarajevo by a team of pediatric surgeons. The surgical procedure was performed after the diagnosis of dermatitis (clinical examination revealed necrotic changes) from the 3 rd to the 7 th day after the injuries.

The surgical procedure was performed after previous reanimation in general anesthesia. We used the Hamby knife to perform tangential necrectomy and electric dermatitis for car and homografts.

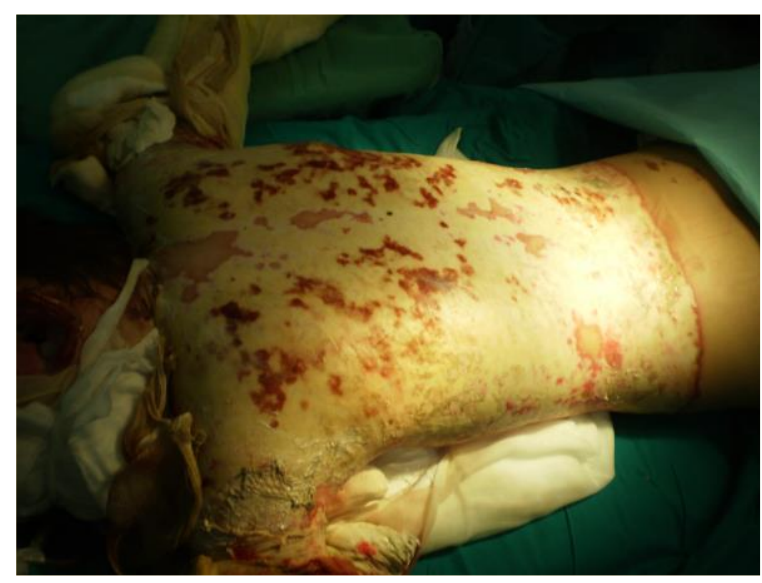

Figure 4: Extensively burnt child by electricity over the $40 \% \mathrm{TBCA}$

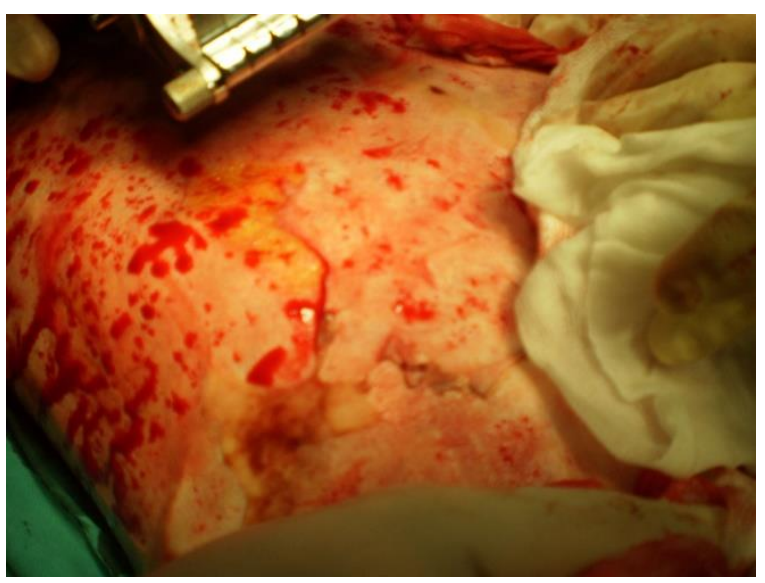

Figure 5: Method of tangential necrectomy

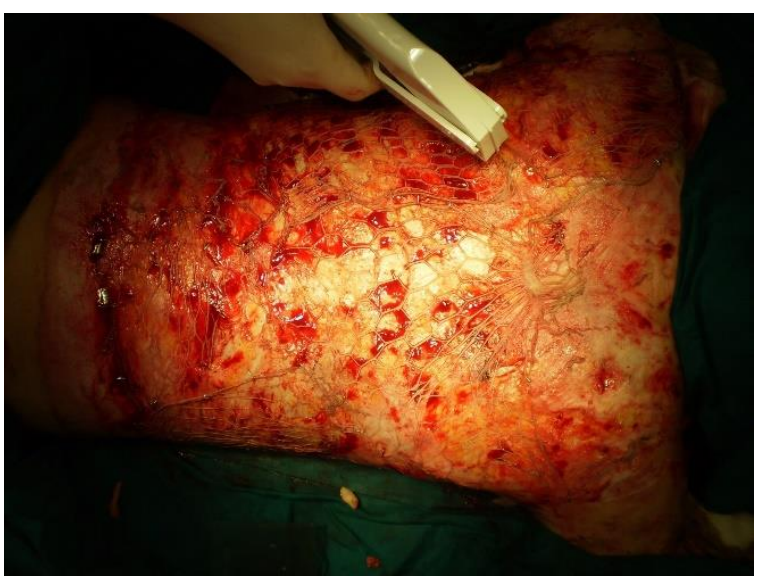

Figure 6: Covering by autotransplantation Thiersch mesh-graft 1:3

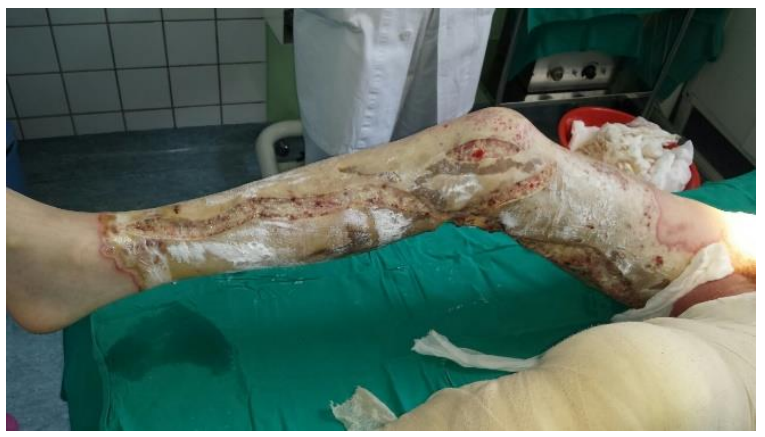

Figure 7: Extensive burns with performed fasciotomy 


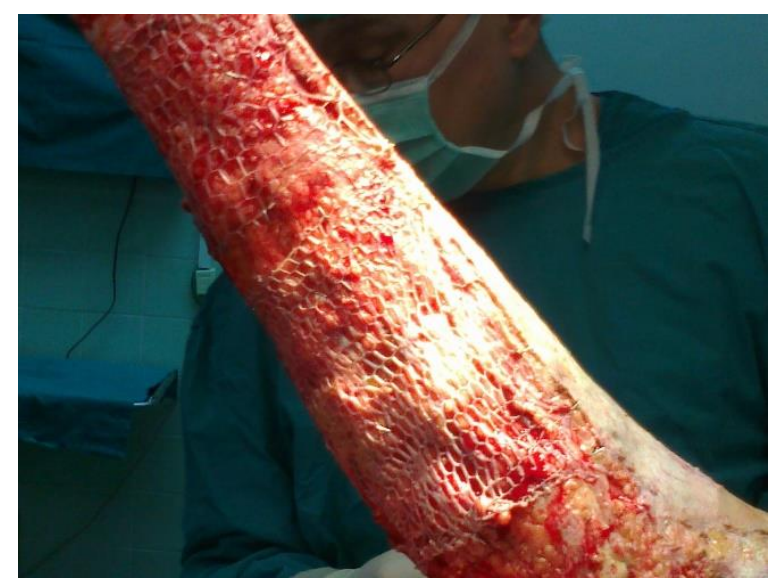

Figure 8: Covering by autotransplantation Thiersch mesh-graft 1:3 after tangential necrectomy

\section{Results}

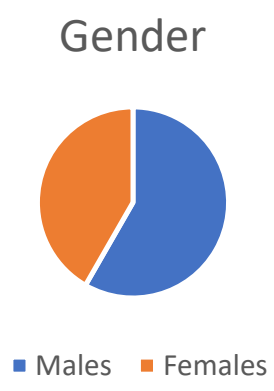

Diagram 1: The gender structure of respondents

Diagram 1 shows the gender distribution of subjects. The boys comprised $58.3 \%$ of the respondents and the girls were $41,7 \%$ of the respondents.
The results presented in Diagram 2 at 3 show that the body temperature was significantly higher in control compared to the test group $(\mathrm{p}<0.001)$.

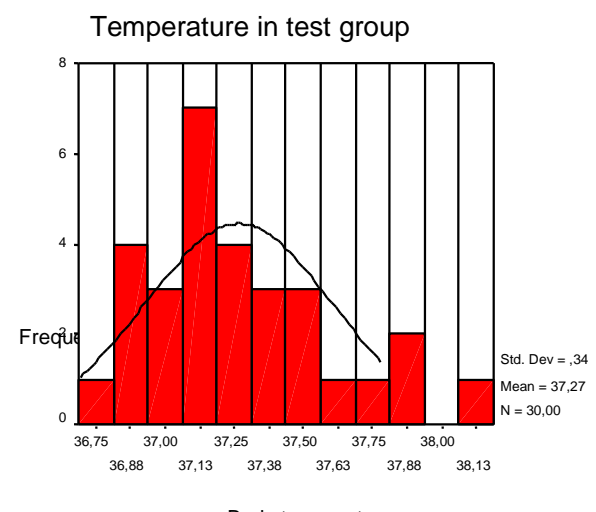

Body temperature

Temperature in control group

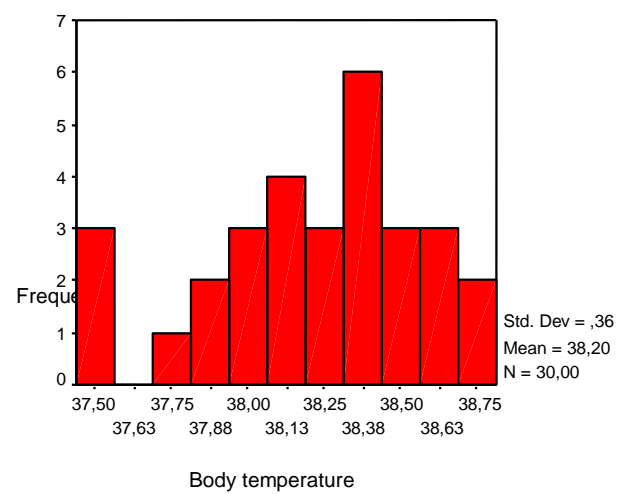

Diagram 2 \& 3: Statistical analysis of the parameters physical axillary temperature.

The mean values $(X+S E M)$ of the body temperature of the examined and the control group were presented:

- the study group $(\mathrm{N}=30)$

- control group $(\mathrm{N}=30)$

The results presented show that the body temperature was significantly 
higher in control compared to the patient group $(\mathrm{p}<0.001)$.

The values shown in Diagram 4.and 5. show significantly lower hematocrit values in the control group compared to the study group $(p=0.002)$.
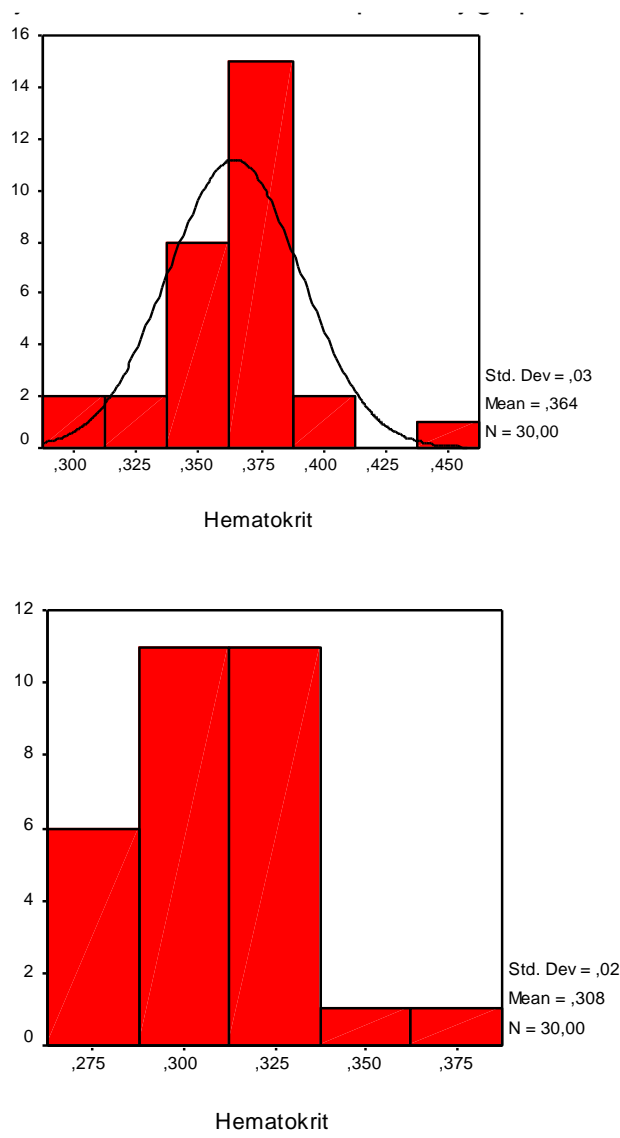

Diagram 4 \& 5: Comparison of hematocrit values from the control and study group.

The values of the hospitalization length in the observed groups are shown in Diagram 6 and 7. The results in show that hospitalization lasted considerably longer in patients in the control group than in the examined group $(\mathrm{p}=0.003)$.
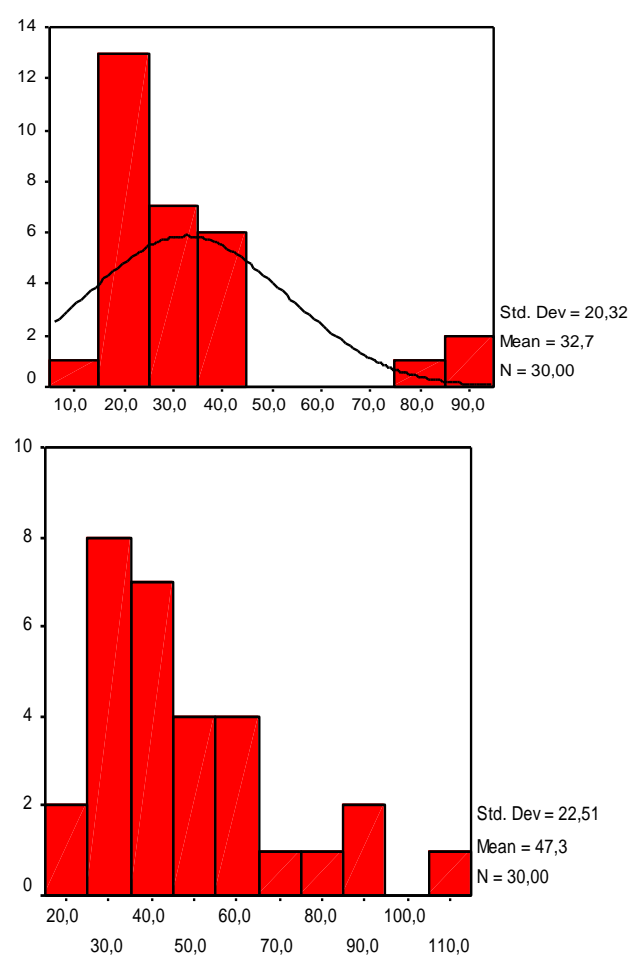

Diagram 6 \& 7: Comparison of the duration of hospitalization between the control and the examined group.

The results of the presence of bacterial infection in the swelling of the burn wound in the observed groups are shown in Table 1.

\begin{tabular}{|c|c|c|c|c|c|}
\hline \multicolumn{5}{|c|}{ Number of isolated bacteria } & \multirow[t]{2}{*}{ Total } \\
\hline & & 0 & $\begin{array}{c}\leq 2 \\
\text { bacteria }\end{array}$ & $\begin{array}{c}\geq 3 \\
\text { bacteria }\end{array}$ & \\
\hline Group & $\begin{array}{l}\text { Examine } \\
\text { d group }\end{array}$ & 5 & 18 & \begin{tabular}{|l|}
7 \\
\end{tabular} & 30 \\
\hline $\mathrm{s}$ & $\begin{array}{l}\text { Control } \\
\text { group }\end{array}$ & 3 & 8 & 19 & 30 \\
\hline Total & & 8 & 26 & 26 & 60 \\
\hline
\end{tabular}

Table 1: Statistical analysis of the presence of bacteria in the abrasion of the burns of the investigated groups. 
Statistical analysis of the microbiological swabs of the early-examined and control group was presented.

- patients in the examined group $(\mathrm{N}=30)$

- control group patients $(\mathrm{N}=30)$

- p < probability

The results presented in Table 17 show that the incidence of bacterial infection of the burns is significantly higher in control compared to the examined group of patients $(\mathrm{p}=0.007)$.

Number of isolated bacteria within the examined group of patient

The most commonly isolated bacteria in the study group was Staphylococcus aureus $(15 / 30$ or $50 \%$ of all subjects). Acinetobacter spp. was isolated in 9 patients (30\% of respondents). Serratia spp. and Enterococcus Foecalis were isolated in 6 patients (20\%). Pseudomonas aeruginosa was isolated in 3 patients $(10 \%)$. Three patients $(10 \%)$ did not isolate any bacteria during hospitalization. In most patients in the study group two or more bacteria were isolated $(13 / 30$ or $43.3 \%)$ during hospitalization. Associated (polymicrobial) infections with three or more microbes were detected in 10 patients $(33 \%)$.

$2 / 30(6.6 \%)$ of patients in the control group did not have a burn infection during hospitalization. The most commonly isolated bacterium was Staphylococcus aureus (18/30 or $60 \%)$. Pseudomonas aeruginosa was isolated in 10 patients $(33.3 \%)$. Acinetobacter spp. was isolated in 9 patients (30\%). In 10/30 patients $(33.3 \%)$, three different microbes were isolated during hospitalization. In the $7 / 30$ patients $(23.3 \%)$, four different microbes were isolated during hospitalization.

The table 2 shows values of body weight loss of the examined groups.

\begin{tabular}{|c|c|c|c|c|c|c|}
\hline & & $\begin{array}{c}\text { Categor } \\
\mathrm{y}\end{array}$ & $\mathrm{N}$ & $\begin{array}{c}\text { Observ } \\
\text { ed } \\
\text { Prop. }\end{array}$ & $\begin{array}{l}\text { Test } \\
\text { Prop. }\end{array}$ & $\begin{array}{l}\text { ignificanc } \\
\text { e (two- } \\
\text { tailed) }\end{array}$ \\
\hline \multirow{2}{*}{$\begin{array}{c}\text { Body } \\
\text { weight on } \\
\text { admissio } \\
n\end{array}$} & $\begin{array}{c}\text { Group } \\
1\end{array}$ & $<27 \mathrm{~kg}$ & 35 & ,59 & ,50 & 0,193 \\
\hline & $\begin{array}{c}\text { Group } \\
2\end{array}$ & $>27 \mathrm{~kg}$ & 24 & 41 & & \\
\hline \multicolumn{3}{|c|}{ Total } & 59 & 1,00 & & \\
\hline \multirow{3}{*}{$\begin{array}{c}\text { Body } \\
\text { weight at } \\
\text { discharge }\end{array}$} & \begin{tabular}{|c|} 
Group \\
1 \\
\end{tabular} & $<25 \mathrm{~kg}$ & 33 &, 55 & ,50 & 0,519 \\
\hline & $\begin{array}{c}\text { Group } \\
2\end{array}$ & $>25 \mathrm{~kg}$ & 27 & ,45 & & \\
\hline & Total & & 60 & 1,00 & & \\
\hline
\end{tabular}

Table 2: Binomijal test

McNemar's test. There is no significant difference in the body weight of admittance and discharge between the examined and the control group of the patients.

The ANOVA test did not show any significant differences within the investigated groups and categories.

\section{Discusion}

Most of the pediatric burns were caused by hot liquids (64.4\%), hot oil (14.8\%), flame $(12.4 \%)$, electrical current $(4,4 \%)$ and contact (3.2\%) (27). The largest 
number of children was between 2-3 years $(42 \%)$. Brogowski $K$. et al. investigated the effect of the cause of the burn on the length of the hospitalization and concluded that the depth of the burn and therefore the length of the hospitalization depends on the cause.

Our research has shown also the following percentages: hot liquids as a cause $73.3 \%$, flammable $23.3 \%$ and contact burns $3.3 \%$. Also, our research has shown a significant association between burns and hospitalization length $(p=0.04)$, hot liquid as a causative agent was significantly more frequent in patients who were shorter hospitalized (<33 days) and flame significantly more frequently appeared as a cause in patients who were longer hospitalized (> 33 days). Further statistical analysis of the mentioned data, ie post-hoc analysis, concluded that there was a significant difference in the length of hospitalization due to the cause of the burn (the flame as the causer causes longer hospitalization). These results can be explained by earlier findings that coagulant necrosis is formed at temperature between 44 and $51 \mathrm{C}$, and above that the protein extraction doubled for each step and that the temperature of the blasted metal and the open flame is over $70 \mathrm{C}$. Also, in our research we concluded that hot liquids significantly more frequently appear as causative agents in patients under 3 years of age while open flame is significantly more frequent in patients older than 3 years $(p=0.04)$.

Z. Janžekovič et al. concluded in their research that the absorption of toxic products produced by the development of bacteria can lead to generalized disease (burn toxemia), leading to the disease and constituting one of the major causes of the mortality of extensively burned patients. By applying careful, gradual, tangential excision, it is possible to eliminate the smallest of the particles of devitalized tissue. The authors have found that profuse bleeding of the vulnerable surface is the best indicator of the vitality of the layer as well as the caliber of blood vessels that make possible a diagnosis of deep dermatitis (28).

Shanin et al. concluded in their research that the morbidity and length of hospitalization was drastically reduced in patients treated with early tangential necrectomy in comparison to patients who were conservatively treated and whose defects were covered only when granulations were advanced (29).

Barrett et al. have revealed that they have achieved remarkable results in survival and reduction of morbidity by performing the tangential necrectomy early in the first 24 hours and at the same time taking full skin biopsy for the epidermal autografted culture technique. The authors have shown that by the method of tangential necrectomy in the first 24 hours, and by covering autograft and taking epidermal culture biopsy, they achieved remarkable results in the prevention of multiplicity and organ response, significantly reduced the duration of treatment and significantly reduced morbidity and mortality (30). Matthew B. Klein et al. have found in their research the benefits of timely surgical treatment for extensively trained patients. In their works, the authors compared the patients in whom the eschara was left intact over the burning surface. Authors believe that proteolytic 
enzymes produced by migrating neutrophils and bacteria within the contaminated eschara cause natural separation of the eschara from the wound bed. After falling of the eschara, wound granulations would serve as a substrate for the graft. The authors argued that surgical treatment was delayed by allowing time to determine which wound he could spontaneously swallow and which required skin graft coverage. The authors have found that in cases of extensive burns, postponement of treatment may lead to more extensive bacterial colonization, increasing the possibility of multiple, organic response and eventually death (31).

Heimbah D. has proved with colleagues that early excision of burns and closure improves survival, reduces infection rate, and reduces length of hospitalization. It also reduces the onset of SIRS (systemic inflammatory syndrome) and the disease. The authors started with surgery on the 3rd day and continued at 2-3 days intervals until all eschara was removed (32). Authors believe that early burn excision and coverage, show improvement in survival, decrease in infection rate, and decrease in hospitalization length. Early deep scarring excretion also shows a reduction in the formation of a hypertrophic scarring. Covering the burn wound is the ultimate goal of treatment of burns. Coverage may be presented by spontaneous epithelization or by covering with the grafts. Sorensen and authors analyzed the Burns Unit Copenhagen series in 1962-1971 (2,460 acute burn victims) and in the period 1972-1975 (660 patients with acute burns), the exposed method of burning best results in cases of superficial burns, and the same method gives the best results in cases of deep burns on the 14th day after the burn. A study by Sorensen et al. shows that surgical excision is desirable in the earliest possible time after a burn incident (during the shock phase) and in small and extensive burns. In cases of deep burns, best cosmetic results are achieved by tangential excision. The authors pose a dilemma when surgical interventions are to be made, whether in shock or 14 days after burns. The authors have achieved the best results in length of hospitalization, reduction of mortality and morbidity and the best cosmetic results in a group of patients operated after 14 days after injury (33).

Our study investigated the influence of early tangential necrectomy on the general state of the patient and showed significantly lower serum protein values in patients who were not surgically treated for the first seven days $(\mathrm{p}<0.001)$. However, we did not find any significant differences between mean body weight gains at admission and postponement of patients treated with two different methods.

Research by Demling R.H et al. has shown that during the first week there is a profound hyper-metabolism that is characterized by an increase in metabolic levels and which in case of severe burns is twice as large as normal. The authors concluded in their research that due to hypermetabolism the regulation of body temperature was changed and resulted in subfebrilation (22).

Edlich et al. have suggested in their research that extensive burns can double the level of metabolism. Body 
temperature reaches values over $38.5^{\circ} \mathrm{C}$ 5 to 15 days after burns and at over $60 \%$ the central temperature was increased up to 2 months due to direct stimulation of hypothalamus with inflammatory mediators and cytokines. Edlich et al have concluded that coverage of the cleaved surfaces reduced by significantly body temperature values due to lowering metabolism and suppressing the delivery of inflammatory mediators to the hypothalamus (34).

Our study compared patients who were surgically treated with the early tangential necrotomy method in the first seven days with patients who were conservatively treated. Body temperature values were significantly higher in patients treated conservatively and postponed surgically than patients treated with early tangential necrotomy ( $p<0.001)$. Most authors reported similar results, explaining them by lowering the basal metabolism value as well as by removing the negative effect of eschara as an excellent basis for bacterial infection. Further, our research shows that patients with a higher number of isolated bacteria had significantly higher body temperature $(p=0.003) \quad$ Our research has shown that the incidence of bacterial infections of burns is significantly higher in patients who were extensively infected, who were not surgicaly treated in the first seven days $(p=0.007)$ compared to patients treated surgically for the first seven days. Further statistical analysis showed that there is a significant association between the length of hospitalization and the number of isolated bacteria from burns $(p=0.002)$. The aim of our further research was to investigate the correlation between the number of bacteria isolated from the burning scorch and the serum hemoglobin values. We have found, in the results, that there is an inverse correlation between the number of bacteria isolated from the burning scorch and the serum values of hemoglobin. ( $\mathrm{rs}=-0.436)$, ie increasing the value of the bacteria in the burn-out of the burn decreases the serum hemoglobin value. In addition to the hemoglobin values, we have established a correlation between the number of isolated bacteria and serum hematocrit values and found that there is a negative association between these values $(p=$ $0.007)$, ie increasing the value of the bacterium reduces the serum hematocrit value.

Lari A.R et al. studying a series of heavily trained patients have shown that Pseudomonas, especially Pseudomonas aeruginosa, is the most dangerous and most pathogenic microorganism. In further research, the authors have suggested that the pre-selection of gram positive bacteria present at an early stage goes into the pre-selection of gram negative bacteria 4-10 days after the injury. In further research, Cremer, Lowbury et al. show a high level of resistance to Pseudomonas aeruginosa to the most commonly used antibiotics. In the investigations of the authors mentioned, the second most commonly isolated microorganism is S. Aureus $(35,36)$.

In the series of patients, the most commonly isolated bacteria were: Staphylococcus aureus, Pseudomonas aeruginosa, Enterobacter cloacae, Klebsiella pneumonia, Enterococcus 
faecalis, Acinetobacter baumannii, Aspergillus species, Candida albicans. Our results show more frequent Staphylococcus aureus $(50 \%)$ while Pseudomonas aeruginosa in the second place is $10 \%$ in the examined and $33.3 \%$ in the control group. There was no significant difference in the presence of bacteria between the control and the examined group.

Numerous studies have shown that extensively burned patient presents a severe disruption of homeostasis. Studies of Loebl and the author, with radioactive $\mathrm{Cr} 51$ and $\mathrm{P} 32$, which investigated half-life of erythrocytes in hard-labored patients, showed that after the first phase characterized by increased hematocrit and blood viscosity, rapid erythrocyte decomposition and erythrocyte abnormalities were observed. The authors concluded that severely burned patients become severely anemic 4th day after burn injury. S.F.Wallner in his papers considers that the main cause of anemia in thermal injuries is not thermal erythrocyte damage but that these are microangiopathic mechanisms and plasma erythropoiesis inhibitor that directly divides into erythrocyte cells. Progressive red blood cell deficiency can be followed by the appearance of multiple abnormal erythrocyte forms in peripheral blood. Morphological changes include microspherocytosis, "associated cells," wild cells, and schitocytes $(17,18)$. Desai et al have shown that early excision significantly reduces intraoperative blood loss and stabilizes the hemogram (37). The results showed that the method of early tangential necrectomy of extensive burned patients also improves the blood image value compared to patients who did not apply this method, serum hemoglobin values were significantly lower in patients who were not surgically treated in the first 7 days ( $p$ $<0.001$ ). Our results obtained by comparing the values of serum hemoglobin with the length of hospitalization showed significantly lower hemoglobin values in patients who were longer hospitalized ( $p<0.003)$. Our research has shown a significant association between body temperature and serum hemoglobin values ( $\mathrm{p}<0.001)$. Also, by testing hematocrit values, we concluded that pathological values of hematocrit were more common in patients who were not operatively treated for the first 7 days $(p=0.002)$. The results of the study examined the effect of length of hospitalization on hematocrit values and we found that patients with pathologic values of hematocrit had significantly longer hospitalization $(\mathrm{p}=0.002)$. Further statistical analysis of data obtained showed a significant positive correlation between hematocrit and hospitalization length $(p=0.003 \mathrm{rs}=0.380)$

Housinger T.A. et al. studied changes in peripheral blood and tried to correlate them with the occurrence of both the survival rate and the degree of survival. The authors compared the group of pediatric survivors and patients who did not survive. They noted that significantly lower platelet counts below $0.1 \times 1012$ / 1 were observed in all untreated patients and concluded that the platelet count is a predictor of the sepsis or death outcome (38). 
Our results showed that there was no significant difference in the serum values of the platelet count and the control group.

The time of surgical intervention: when to start early tangential necrectomy - is the subject of research by many authors. In 1966, S.R.Lewis described over 1000 cases by citing as time for necrectomy and covering the graft period before 24 and 25 days. The authors of D.M.Jackson and Z.Janžekovič in separate studies conclude that after the first phase of burn-out shock characterized by coagulant necrosis, dilated capillaries and increased permeability of blood vessels, demarcation between vital and ireversibly destroyed (necrotic) tissue becomes apparent. In the second phase the organism reacts to the necrosis with the inflammatory reaction that can cause micro-thrombosis and secondary necrosis of deeper, vital dermis layers. In this period (the first 3 days) resuscitation is completed, the reanimation carried out, the authors believe that the period from the 3rd to 5th day after the injury is most ideal for the implementation of the operation. The authors explain that after the first phase of burn-out shock characterized by coagulant necrosis, dilated capillaries and increased permeability of blood vessels, the demarcation between vital and ireversibly destroyed (necrotic) tissue becomes apparent. Experiences of Janžekovič et al in the works show that the appearance of the surface (black and brown fields, white, gray-yellow, marbled development of the surrounding erythema, occurrence of ash) and application of pin prick test is sufficient to determine the depth of the burn with great certainty. In the second stage the organism reacts to the necrosis of inflammatory reaction that can cause micro-thrombosis and secondary necrosis of deeper, vital dermis layers (39). To ensure the degree of safety of depth determination and thus the correct indication of operative treatment, recent authors' works demonstrate the advantages of the following procedures in determining the exact depth of the injured wound. Kahn published the experiences with burn biopsy (40), Kalus and Warchel used ultrasound and LDPM (laser doppler perfusion monitoring) (41). Essex et al have published achievements and high accuracy in determining skin involvement with LDI (laser Doppler imaging) (42). While Albsjom was rejuvenated by LDF (laser Doppler flowmetry) (43). The use of fluorescein fluorescence photos was published by Zuckerman et al (44) while Celicioz with methylene blue was able to more accurately determine the depth of burn (45).

Magliacani et al., in their diagnostic studies, also introduced biopsy as a method (33). Histological monitoring of the involvement of tissue infection confirms the effectiveness of targeted antiseptic therapy or suggests alternative techniques. Progressive tissue involvement with infection, associated with increased bacteria, is a clear indication for more energetic target therapy. The authors concluded that the concept of early escharectomy of heavily burned patients must be associated with emergency necrotomy when a bacterial invasion of vital tissue under the eschar is present, and using earlier knowledge 
of colonization of bacterial burns authors suggest 4 th or 5 th day as a timely excision.

Still et al. published experiences with tangential necrectomy performed in the first $48 \mathrm{~h}$ after injury compared to patients who were operated after the first $48 \mathrm{~h}$ (47). Patients who were operated after the first $24 \mathrm{~h}$ after admission had a statistically significant increase in length of stay in hospital compared to patients who were operated in the first $24 \mathrm{~h}$. In their study mortality and morbidity as well as the need for postoperative operations are not significantly different between the two groups.

Further results of our research are not in accordance with the foregoing. Due to the technical limitations and the inability to determine the depth of the burn through modern methods we have been forced to resort to classical methods of determining the depth of the burn. In the first $24-48 \mathrm{~h}$ we were not able to determine the depth of the burn and thus compare our results with the abovementioned results. In study on experimental animals, the authors found that the blood flow in the burn early showed a marked increase in the 4th day after the injury, reaching its peak between 5 and 14 days. Further research has shown that the rise in blood flow with paralelically developed inflammation of the wound occurs in vital tissue under the eschara. Significant increase in blood loss should be expected with the excision of the wound after 5-6 days. For these reasons, the authors recommend that excision should be done before day 5 to avoid increased blood loss.
Our research is similar to Janžekovic's and authors' research since we achieved the best results by early tangential excision within 5.-6. postoperative day.Colonization of the wound develops during the first week and manipulation by colonized or infected burns increases the risk of bacteriemia. Pallua N.et al proved that sepsis is the most common cause of mortality in burn injuries. The main source of bacteria that causes sepsis is the burn wound itself. The authors evaluated whether current necrectomy compared to early necrectomy leads to decreased septic complications and posttraumatic lethality. They evaluated pediatric patients extensively digested. Lethality was significantly reduced in the group where the early tangential necrotomy was done in the first 3 days, compared with the group where it was done after the 3rd day $(p<0.01)$. In this study, it is demonstrated that current necrotomy compared to early necrectomy in young patients results in a significant reduction in lethality and lethality in severe thermal burns (48). The degree of extensibility of burns, the impact of burns on morbidity and mortality was the subject of the research of the following authors. Thompson and colleagues in their studies investigated patients extensively burned, 2nd and 3rd degree (49). Over $30 \%$ of patients were treated with early tangential necrosctomy and reported a significant mortality decline, hospitalization lengths were not considered in their series. MacMillan and Artz discussed in 1957 patients with large burns that had been excised and an improvement was found in the length of hospitalization between survivors (50). Herndon et al found no 
changes in mortality among adult patients burned over 30\% treated with early excision (51). Scott-Conner et al. investigated the wound closure index, found that excision over $20 \%$ improved survival (52). Compared to 11 US units, Wolfe et al have shown that faster coverage of deep burns is associated with lower mortality (53). Chicarilli et al published scores of patients burned over $30 \%$ of TBSA and showed significant shorter hospital stay and improved survival after aggressive excision (54). Tchervenkov et al. have published improved neutrophil function in experimental animals with early excision (55).

Research by Sheridan et al has shown that children under 2 years do not tolerate thermal injury as older children. Morbidity and mortality in these patients was higher in their studies than in older children. One of the most important reasons is, according to the authors, immature immune systems and increased need to compensate for fluid leading to greater risk of hypovolemic shock and infection with smaller children. S.E.Morrow noted in subsequent studies that there was a significant difference in patients up to 4 years of age compared to older patients in response to thermal injury.

Our research did not show a significant difference in morbidity among patients aged younger than 4 years and older than 4 years $(p=0.36)$.

Maurage C., et al, analyzed 850 children hospitalized for a period of 10 years, with a mean duration of hospitalization of 30.8 days, while for patients over $25 \%$ of TBSA the average length of hospitalization was 63.6 days (56) .
Our results showed that the length of hospitalization of patients with extensive lesions that were conservatively treated and postponed surgically in relation to the patients treated for the first seven days was significantly longer $(p=0.003)$. Our research has shown that patients whose size of extensive burns exceeded $25 \%$ of the total body surface area were significantly longer hospitalized compared to patients whose burned body surface area was below $25 \%$ ( $p=$ 0.016 ).

The allowed size of the area that can be removed in one act was the topic of many authors' discussions.

Magistrates et al in their research have concluded that more than $15-20 \%$ of necrotic tissue should not be removed in one act (23). The surface area over $25 \%$ should be covered with autografts using mesh-graft $1 / 2$. If over $25 \%$ of burns, authors suggest substitutions: biological, biosynthetic and synthetic.

Edlich and Desai et al., concluded in their papers that the gold standard of patients was extensive-burn early excision and graft coverage. The mortality of patients with massive burns was significantly reduced by early tangential excision of complete burn wound. The authors concluded in their papers that these surfaces should be covered by autograft from healthy regions of 1: 1 or 2: 1 for burns less than $30 \%$. In the case of burns larger than $40 \%$ TBSA, skin autografts should be 3: 1 or 4: 1 meshes, possibly associated with a cadaver allograft used temporarily to cover the remaining open vulnerable surfaces. In their research, they have found that the cadaver allograft has become valuable to the vulnerable surface and represents a 
partial barrier to infection but may also transmit antigen and infectious disease (55). Investigations by Burton. $\mathrm{H}$ and Tompkins have shown that the best results are achieved by burns over 30\% using mesh-grafts of 1.5: 1 or 2: 1 and in the case of burns over $40 \%$ by partial necrectomy (up to $20 \%$ every other day) and by covering vulnerable regions with autografts mesh 1.5: 1, 2: 1 or 3: 1 or autografts $3: 1$ with alografts or synthetic substitution of the cleaved surface $(57,58)$.

Janežic A., and Herndon published routine use of allografts, xenografts, or artificial coverings with Integra, Dermagraft-TC, and over $40 \%$ TBSA $(59,60)$.

In our research, we concluded that, with regard to blood loss, it is possible to perform necrectomy and covering with mesh-graft 1: 1 or $1: 2$ to $30 \%$ of the burn surface in children. In the case of burns over $40 \%$, we combined auto transplantation with homotransplantation of the skin of parents or close relatives.

\section{Conclusions}

The method of early tangential necrectomy of extensively burned children significantly affects morbidity and mortality (improves the general condition of a patient, improves laboratory parameters, reduces the chance of early infection, reduces the length of hospitalization).

For children with burns that take over $30 \%$ of the total body surface area, early tangential necrectomy should be applied to a surface of about $20 \%$ of the total body surface area as prevention of blood loss due to necrectomy and autotransplants.

For children with burns that take over $40 \%$ of the total body surface area, taking homotransplant from parents or close relatives reduces morbidity and mortality.

The most optimal time to perform the early tangential necrectomy is between 4 and 6 days after the injury.

\section{References}

1. Demling R.H.Lawrence W.Way. Opekotine i druge termičke povrede.Hirurgija, savremena dijagnostika i liječenje.1990.;26077.

2. A.R.Moritz,F.C.Henriquez. Studies of thermal injury II.The relative importance of time and surface temperature in the causation of cutaneous burns. Am J Pathol 1947;23:695-720.

3. D.M.Jackson. The Diagnosis of the Depth of Burning.British Journal of Surgery 1953.40:588-96.

4. J.V.Boykin,E.Eriksson,R.N. Pittman. Microcirculation of scald burn:an in vivo experimental study of the hairless mouse ear.Burns 1980;7:335-8.

5. G.S.Johnson,T.Lineberger,A.L.Ho them,K.Adams,T.Hikson,W.Wink ewerdwe. Erythrocyte flexibility in the burned patient.1979;6:91-5. 
6. B.E.Zawacki. The local effects of burn injury. The Art and Science of Burn Care.Rockville,MD:Aspen,1987:2 9.

7. B.A.B.A.Pruitt,J.A.Moncrief. Current trends in burn research.J Surg Res 1967;7:280-93.

8. C.Jelenko,J.M.Ginsburg. Water holding lipid and water transmision through homeothermic and poikilothermic skins.Proc Soc Exp Biol Med 1971;139:1059-62.

9. F.P.Underhill The significance of anhydremia in extensive surface burn.JAMA 1930;95:852-7.

10. F.D.Moore The body-weight budget:basic fluid therapy for the early burn.Surg Clin North Am 1970;50:1249-65.

11. C.P.Artz,J.A.Moncrief The burn problem.In Artz The treatment of Burns.Philadelphia:W.B.Saunders ,1969:1-22.

12. B.A.Pruitt,A.D.Mason,J.A.Moncri ef Hemodynamic changes in the early post burn patients:the influence of fluid administration and of a vasodilator (hydralazine).J Trauma 1971;11:36-46.

13. L.Leape Initial changes in burnes:tissue changes in burned and unburned skins of Rhesus monkeys.J Trauma 1970;10:48892.

14. W.W.Monafo The treatment of burn shock bytheintravenous and oral administration of hypertonic lactatedsalinesolution.J Trauma 1970;10:575-86.
15. C.R.Baxter Problems and complications of burn shock resuscitacion.Surg Clin North Am 1978;58:1313-22.

16. R.H.Demling Fluid resuscitacion.The Art and Science of Burn Care.Rockville:Aspen,1987:189202.

17. S.W.Merrel,J.R.Saffle,J.J.Sullivan, P.D.Navar,M.Kravitz,G.D.Warde $\mathrm{n}$ Fluid resuscitacion in thermally injured children.Am J Surg 1986;152:664-9.

18. T.A.Graves,W.G.Cioffi,W.F.McM anus,A.D.Mason,B.A.Pruitt Fluid resuscitacion of infants and children with massive thermal injury.J Trauma 1988;28:1656-9.

19. H.Rode,D.M.Heimbach Surgical treatment of thermal injuries.1990.830-8.

20. Janzekovic Z: A new concept in early excision and immediate grafting of burns. J Trauma 1970; 10:1003-1008

21. KucanJ.Burns.PlasticSurgery:Indi cations, Operations and Outcomes Ch 134 2002.2385-97.

22. Derganc M. Introduction to the Symposium.Present Clinical Aspects of Burns-A Symposium,Maribor 1968. 13-21.

23. O.Marinković,D.Bajec,P.Radojko vić. Results of Treatment of Burns in Children with Silver Nitrate Solution Method.Present Clinical Aspects of Burns-A Symposium Maribor 1968.75-9.

24. E.J.Lowburn. Infection in Burns. British Journal of Plastic Surgery.1967.;211-21 
25. D.M.Jackson,P.A.Stone.Tangentia 1 Excision and Grafting of BurnsThe Method and a Report of 50 Consecutive Cases.British Journal of Plastic Surgery.1972.25, 416-26.

26. G.Magliacani The Surgical Treatment of Burns:Skin Substitutes.Annals of the Mediterranean Burns Council,vol 3.1990.220-35.

27. Zaidi M.M., Abusetta A., Brogowski K, Agrawal P.L., Franka M.R. Analysis of burned children treated in the burns and plastic surgery center, Tripoly Libya, in the year 1992Annals of Burns and Fire Disasters - vol. X n. 1 - March 1994.

28. Z.Janžekovič The Dermal Burn.Present Clinical Aspects of Burns-A Symposium Maribor 1968.215-22.

29. Shahin A., Shadata G., Franka M.R., Abusetta A., Brogouski A., Ezzaidi Complications of burns in children - a study of 266 sverely burned children admitted to a burns centre Annals of Burns and Fire Disasters - vol. XI - n. 1 March 1988.

30. Barret J.P., Wolf S.E.,Desai M.H., Herndon D.N. Total burn wound burn excision of massive paediatric burns in the first 24 hours post-injury Annals of Burns and Fire Disasters - vol. XII - n. 1 March 1999

31. Matthew B. Klein, MD; David Heimbach Menagement of the Burn Wound ACS Surgery Principles 2006.
32. Heimbach D, Early burn excision and grafting:Surg Clin North Am 1987 Feb;67(1):93:107.

33. Sørensen S. Early tangential necrectomy, Jurn Plast.Surg ,2005.44 (2):23:191.

34. Engrav LH,Edlich et al: Early excision and grafting vs nonoperative treatment of burns of indeterminent depth:A randomized prospectiver study.J Trauma 1983; 23:1001.

35. Cramer K,Lowbury E. et al: Local prophylactic chemoterapy for burns. Lancet 1992.2,958-963.

36. D'Alesandro MM; Gruber DF Quantitative and functional alterations of peripheral bloodneutrophils after $10 \%$ and $30 \%$ thermal injury. D'Alesandro MM; Gruber DF Journal of burn care \& rehabilitation [J Burn Care

37. Rehabil] 1990 Jul-Aug; 11 (4), pp. 295-300.

38. Desai MH, Herndon DN, Broemeling L, et al: Early burn wound excision significantly $\mathrm{r}$ e duces blood loss. Ann Surg 1990; 211:753-762

39. Housinger TA,Brinkerhoff C.,Warden GD. The relationship between pllatelet count, sepsis, and survival in pediatric burn patients. Archives of surgery 1993. 128(1) 65-7.

40. Z.Janžekovič.Consistent Application of Generally Adopted Surgical Principles in the Treatment of the Burn Wound.Present Clinical Aspects of Burns-A Symposium Maribor 1968.100-12. 
41. A.M.Kahn,V.L.McCrady,V.J.Rose n:Burn Wound Biopsy. Scand.J.Plast.Reconstr. Surg.,1979.13;53.

42. A.M.Kalus.Application of Ultrasound in Assessing Burn Depth.20.Lancet,1979. 28:188.

43. T.J.H.Essex, P.O.Byrne.A Laser Doppler Scanner for Imaging Blood Flow in Skin. J.Biomed.Eng.,1991.13.189.

44. B.Albsjom,J.Micheels,B.Sorensen.
Laser Doppler
Flowmetry
Measurements of Superficial
Dermal,Deep Dermal and
Subdermal
Burns.Scand.J.Plast.Reconstr.Surg . 1984.18:75.

45. A.Zuckerman.Fluorescin

Fluorescence Photography for the Evaluation

Burns.J.Biol.Photogr.1983.51:33.

46. B.Celikoz,M.Deveci,A.Nisanci.Ea rly Tangential Excision with the Guidance of Methylene Blue Application.Annals of Burns and Fire Disasters.vol XII 1999. 76-81

47. Di Lonardo A., Ferrante M., Maggio G., Bucaria V., Del Zotti M., Brienza E.Histological assesment of the level of burn wound infection:Diagnostic and therapeutic strategies. Annals of Burns and Fire Disasters.vol II 2004. 52-14

48. J.M.Still,E.J.Law,K.Belcher,D.Thir uvaiyarv.Decreasing Lenght of Hospital Stay by Early Excision and Grafting of Burns.SMJ1996.

49. Pallua N.,Surgical prevention of post-traumatic infection by immediate necrectomy of burn wounds, Langenbecks Arch Chir
Suppl Kongressbd.1996;113:11448.

50. Thompson P, Herndon DN, Abston S, et al: Effective early excision on patients with major thermal injury. J Trauma 1987; 27:205-207

51. MacMillan BG, Artz CP: A planned evaluation of early excision of more than $25 \%$ of the body total in burns. Surg Forum 1957; 8:88

52. Herndon D, Gore D, Cole M, et al: Determinant of mortality in pediatric patients with greater than $70 \%$ full thickness total body surface area thermal injury treated by early total excision and grafting. J Trauma 1987; 27:208212

53. Scott-Conner CE, Coil JA, Conner $\mathrm{HF}$, et al: Wound closure index: a guide to prognosis in burn patients. J Trauma 1986; 26:123127

54. Wolfe RA, Roy LD, Flora JD, et al: Mortality differences in speed of wound closure among specialized burn care facilities. JAMA 1983; 250:763-766

55. Chicarilli ZN, Cuono CB, Heinrich JJ, et al: Selective aggressive burn excision for high mortality subgroups. J Trauma 1986; 26:19-25

56. Tchervenkov JI, Epstein M, Silberstein E, et al: Early burn wound excision and skin grafting post burn trauma restores in-vitro neutrophil delivery of inflamm atory lesions. Arch Surg 1988; 123:1477-1481 
57. Maurage C.,Robert M.,Mercier C.,Billard J. Burns in Children :10 - year experience of the Pediatric Surgical Service of Tours (apropos of 850 cases). J Anesth Analg 1977;34 (6):1303-13.

58. Desai MH, Rutan RL, Herndon DN. Conservative treatment of scald burns in superior to early excision. J Burn Care Rehabil 1991;12:482-4.

59. Tompkins RG, Remensnyder JP, Burke JF et al. Significant reductions in mortality for children with burn injuries through the use of prompt eschar excision. Ann Surg 1988:577-85.

60. Janežič A. Experience with cultured epithelial autografts in the treatment of patients with extensive full thickness thermal injuries Annals of Burns and Fire Disasters - vol. XIV - n. 3 september 2001.

61. Herndon DN, Parks DH. Comparasion of serial debridement and autografting and early massive excision with cadavar skin overlay in the treatment of large burns in children. J Trauma 1986;26:149-52. 